\title{
Investigation of effective media applicability for ultrathin multilayer structures
}

Sukham, Johneph; Takayama, Osamu; Mahmoodi, Maryam; Sychev, Stanislav; Bogdanov, Andrey; Tavassoli, Seyed Hassan; Lavrinenko, Andrei V; Malureanu, Radu

\section{Published in:}

Nanoscale

Link to article, DOI:

$10.1039 / \mathrm{c} 9 \mathrm{nr} 02471 \mathrm{a}$

Publication date:

2019

Document Version

Peer reviewed version

Link back to DTU Orbit

Citation (APA):

Sukham, J., Takayama, O., Mahmoodi, M., Sychev, S., Bogdanov, A., Tavassoli, S. H., Lavrinenko, A. V., \& Malureanu, R. (2019). Investigation of effective media applicability for ultrathin multilayer structures. Nanoscale, 11(26), 12582-12588. https://doi.org/10.1039/c9nr02471a

\section{General rights}

Copyright and moral rights for the publications made accessible in the public portal are retained by the authors and/or other copyright owners and it is a condition of accessing publications that users recognise and abide by the legal requirements associated with these rights.

- Users may download and print one copy of any publication from the public portal for the purpose of private study or research.

- You may not further distribute the material or use it for any profit-making activity or commercial gain

- You may freely distribute the URL identifying the publication in the public portal 


\section{Journal Name}

\section{ARTICLE TYPE}

Cite this: DOI: $10.1039 / x x x x x x x x x$

Received Date

Accepted Date

DOI: $10.1039 / x x x x x x x x x x$

www.rsc.org/journalname

\section{Investigation of effective media applicability for ultra- thin multilayer structures}

\author{
Johneph Sukham, ${ }^{a}$ Osamu Takayama, ${ }^{a}$ Maryam Mahmoodi, ${ }^{b}$ Stanislav Sychev, ${ }^{c}$ Andrey \\ Bogdanov, ${ }^{c}$ Seyed Hassan Tavassoli, ${ }^{b}$ Andrei V. Lavrinenko ${ }^{a}$ and Radu Malureanu*a
}

Multilayer hyperbolic metamaterials (HMMs) are highly anisotropic media consisting of alternating metal and dielectric layers with their electromagnetic properties defined by the effective medium approximation (EMA). EMA is generally applied for a large number of subwavelength unit cells or periods of a multilayer HMM. However, in practice, the number of periods is limited. To the best of our knowledge, a comparison between rigorous theory, EMA and experiments to investigate the minimum number of layers that allow for low error of EMA results has not yet been investigated. In this article, we compared the reflectance response of the effective anisotropic HMMs predicted by the scattering matrix method (SMM) and EMA with optical characterization data, having the unit cell twenty times smaller than the vacuum wavelength in the visible range. The fabricated HMM structures consist up to ten periods of alternating $10 \mathrm{~nm}$ thick $\mathrm{Au}$ and $\mathrm{Al}_{2} \mathrm{O}_{3}$ layers deposited by sputtering and atomic layer deposition, respectively. The two deposition techniques enable us to achieve a high quality HMM with low roughness: the root mean square (RMS) is less than 1 $\mathrm{nm}$. We showed that the multilayer structure behaves like an effective medium from the fourth period onwards as the EMA calculation and experimental results agree well having below $4 \%$ the mean square standard deviation of reflectance (MSDR) for the wavelength range from 500 to $1750 \mathrm{~nm}$ with a wide incident angle range. These results could have an impact on the design and development of active metamaterials applications ranging from imaging to nonlinear optics and sensing.

\section{Introduction}

Hyperbolic metamaterials (HMMs) can play a key role in nanophotonics due to their controllability and tunability of the propagation of light. They are highly anisotropic media which have indefinite or hyperbolic dispersion and their properties are determined by their effective electric permittivity or magnetic permeability. Their main property is that they behave like metals in one direction and dielectric in the other direction in a broadband wavelength range of operation ${ }^{1,2}$. HMMs have shown various phenomena and applications in engineering photonic density of states ${ }^{3}$, enhanced spontaneous decay and recombination rates $^{4,5}$, super lens beating diffraction limit ${ }^{6-8}$, extremely high biosensing sensitivity ${ }^{9}$, polarization selectivity ${ }^{10}$. They can be realized in the visible to mid-infrared wavelength region by a combination of metal and dielectric in the form of multilayers ${ }^{11-13}$,

\footnotetext{
${ }^{a}$ Technical University of Denmark, Dept. of Photonics Engineering, Ørsteds plads, bldg. 345V, 2800 Kgs. Lyngby, Denmark email id - rmal@fotonik.dtu.dk

${ }^{b}$ Laser and Plasma Research Institute, Shahid Beheshti University, Tehran, Iran

${ }^{c}$ Saint Petersburg National Research University of Information Technologies, Mechanics and Optics, 197101, St Petersburg
}

trenches ${ }^{14-16}$, nanorods ${ }^{17}$. The different suitable materials to design HMMs depending on the applications for various wavelength ranges have been studied in detail ${ }^{18,19}$.

$\mathrm{Au}$ is the most practical plasmonic material to fabricate HMMs in the visible and near-infrared wavelength range due to its chemical stability ${ }^{11,20,21}$. However, Au does not adhere well on dielectric substrates and thus the films are fully formed only above 15 $\mathrm{nm}$ when not using any adhesion layers. The percolation threshold limit for Au films ranges from a thickness of around 8 to 15 $\mathrm{nm}^{22}$ depending on the deposition technique used and deposition parameters.

$\mathrm{Ti}$ and $\mathrm{Cr}$ are the standard adhesion layers used for $\mathrm{Au}^{23}$. The influence of $\mathrm{Ti}$ and $\mathrm{Cr}$ adhesion layers on ultra thin $\mathrm{Au}$ films has been studied morphologically using electron microscopy and found that $\mathrm{Cr}$ inter-diffuses within the Au layer forming a $\mathrm{Cr}-\mathrm{Au}$ alloy with a partially oxidized adhesion layer ${ }^{24}$. Moreover, the introduction of a few nanometer thick metallic adhesion layers has shown to increase the broadening of surface plasmon polariton (SPP) resonance due to the extra absorption and scattering from the metal ${ }^{25}$. As an alternative to $\mathrm{Ti}$ and $\mathrm{Cr}$, we recently reported that the silane based adhesion promoter (3-Aminopropyl) 
trimethoxysilane (APTMS) is a better adhesion layer between oxide and Au in comparison with metals regarding support of highly confined surface plasmon modes ${ }^{26}$.

The propagating bulk plasmon modes in HMMs are due to the coupling of short range surface plasmon polaritons (SR-SPPs) in each metal-dielectric interface of the multilayer HMMs. ${ }^{27,28}$. This coupling implies that the electric field is localized at the interfaces, thus making adhesion layer's contribution even more significant. Therefore, using nonmetallic adhesion like APTMS is the favorable option for the fabrication of high quality Au based HMMs.

The electromagnetic properties of HMMs are routinely predicted by EMA, which is generally applied when the vacuum wavelength of light is much larger than the unit cell or period of the hyperbolic metamaterial ${ }^{29-32}$. In ref.32, the authors analyse the applicability of EMA when the number of periods varies and show theoretically that the EMA can be applied only from a certain number of periods. Since we use a ZnSe prism to excite high-k waves in the system, we believe that the limits we find are stricter that the ones obtained in the cited work. However, it is a natural question whether the EMA is applicable on a fabricated structure with a limited number of periods. The minimum thickness or number of periods for a structure sufficient to behave like an effective medium is still unclear. Understanding this limit could help in designing of the HMM structures needed for various applications.

To study the applicability of EMA, we fabricated a set of multilayer structure with various periods of HMMs samples: 1, 2, 3, 4, 6, 8, 10 consisting of $10 \mathrm{~nm}$ thick $\mathrm{Au}$ and $\mathrm{Al}_{2} \mathrm{O}_{3}$ layers respectively, using APTMS as adhesion promoter in between each $\mathrm{Au}$ and $\mathrm{Al}_{2} \mathrm{O}_{3}$ interface. In terms of quality of the fabricated multilayer structure, the HMM with four periods has a RMS roughness of $0.40 \mathrm{~nm}$ and the final RMS roughness of the $10^{\text {th }}$ period sample is $0.80 \mathrm{~nm}$. We characterized the samples optically using the prism coupling scheme to study their reflectance spectra. Experimental results were then compared with EMA calculations. We used the mean square deviation of reflection (MSDR) to compare the reflection difference between EMA with multilayer and experiment using mean square deviation calculation as shown in equation (1) and (2), respectively. Comparison certifies that the reflection spectra in this multilayer case start matching the effective medium predictions from the $4^{\text {th }}$ period having as low as $4 \%$ MSDR.

Although our results are obtained in specific HMM configuration, the general message remains: the EMA has a lower limit of applicability and one should take that into account when using it for describing the behavior of HMMs. This may prove of utmost importance for designing HMM-based devices since their characteristics may differ significantly from the ones calculated using the EMA theory. Most of the applications of HMMs in waveguiding ${ }^{33-35}$, refractive index sensing ${ }^{11}$, optical cavity design ${ }^{36}$, hyperlens for sub-diffraction imaging ${ }^{37}$ and so on are based on EMA. Therefore, it is important to know its range of applicability in order to design the needed devices. If EMA is applied outside its confidence interval, the performance of the HMM structures for these, and other, applications will deviate from the desired ones.

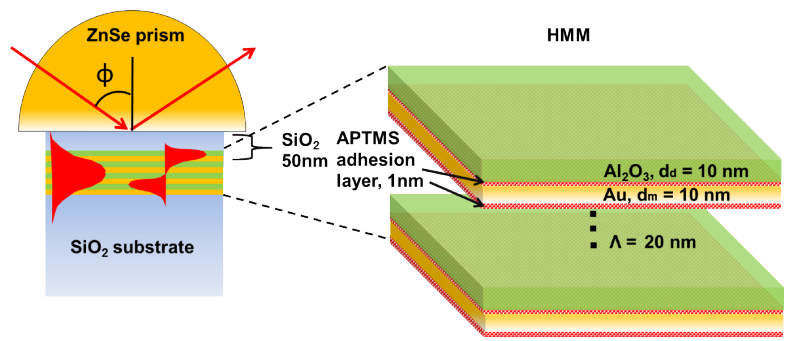

Fig. 1 Schematic illustration of metal-dielectric multilayer structure, excitation and detection of SPPs using the Otto configuration. Symmetric plasmonic waveguide structures are made of $\mathrm{SiO}_{2}$-adhesion layer-Au-adhesion layer- $\mathrm{SiO}_{2}$. The adhesion layers under consideration are $1.0 \mathrm{~nm}$ thick APTMS layers.

\section{Results and Discussion}

\subsection{Theoretical Study}

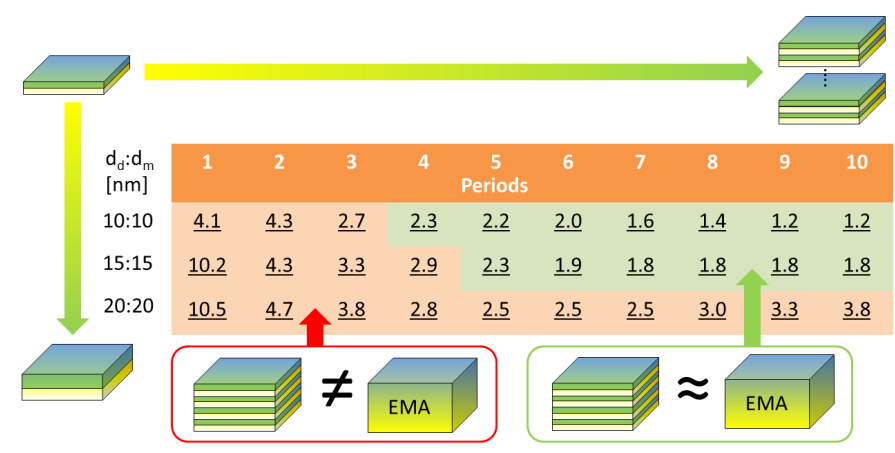

Table 1 Table of mean-square error reflectance, MSDR $<2.5 \%$, between multilayer structures and EMA-based HMM structures. Green shaded area represents MSDR $<2.5 \%$ where EMA approximately holds and red shaded area is where behavior of HMMs are different from EMA.

We numerically studied the influence of the thickness of layers ( $d_{m}$ and $d_{d}, \Lambda=d_{m}+d_{d}$ ) and number of periods on the behavior of multilayer HMMs properties (reflectance) in comparison with the ones predicted by EMA. For simplicity we consider a HMM composed of $\mathrm{Au}$ and $\mathrm{Al}_{2} \mathrm{O}_{3}$ with metal filling factor of 0.5 , without APTMS adhesion layer. Table 1 shows the summary of numerical analysis of reflectance difference between of the multilayer HMM calculated by 1D SMM and 1D EMA. We considered TM-polarized incident light with the magnetic field parallel to the plane of interface to calculate the reflectance. The reflectance calculated by 1D SMM and 1D EMA is based on the model in Figure 1. The details of the procedure are presented in Numerical Simulation section in Method. Here, we define the criteria for the applicability of EMA as the standard deviation of reflectance MSDR $<2.5$ $\%$. The MSDR is defined as

$$
M S D R=\sqrt{\frac{1}{N} \sum_{\phi, \lambda}\left[R(\lambda, \phi)_{\text {multilayer }}-R(\lambda, \phi)_{E M A}\right]^{2}},
$$


where reflectance is a function of two variables, namely angle of incidence and wavelength as shown in equation 1 . The angle of incidence is varied between $27^{\circ}$ and $81^{\circ}$ and the vacuum wavelength spans from 500 to $1750 \mathrm{~nm} . R(\lambda, \phi)_{\text {multilayer }}$ and $R(\lambda, \phi)_{E M A}$ are the simulated reflectance spectra and $\mathrm{N}=57600$ is the number of points used for the simulation. The analysis results shown in table 1 , imply that in the case of $\mathrm{Au}$ and $\mathrm{Al}_{2} \mathrm{O}_{3}$ films of $10 \mathrm{~nm}$ each, multilayer structures behave close to effective medium from 4 periods (total thickness of $80 \mathrm{~nm}$ ). For 15 $\mathrm{nm}$ thick films the applicability criteria is satisfied from 5 periods onwards. In the case of $20 \mathrm{~nm}$ thick layers for $\mathrm{Au}$ and $\mathrm{Al}_{2} \mathrm{O}_{3}$, the HMM structures behave different from EMA counter part and do not satisfy the standard deviation condition MSDR $<2.5 \%$ until at least 10 periods. To be noted that even the generally accepted condition of $\lambda>10 *\left(d_{d}+d_{m}\right)$ is still fulfilled, the EMA condition, as defined by maximum admissible deviation, is not.

Additionally, we observe an unexpected feature of the EMA vs. rigorous theory comparison. For the $20 \mathrm{~nm}$ : $20 \mathrm{~nm}$ case, the MSDR seems to increase after 8 periods, hinting towards the possibility that, for big number of periods, the applicability criteria might not be satisfied. However, this observation is not fully verified. Thereafter, we proceed experimental demonstration with multilayer HMM of $d_{d}: d_{m}=10 \mathrm{~nm}: 10 \mathrm{~nm}$.

\subsection{Experimental Study}

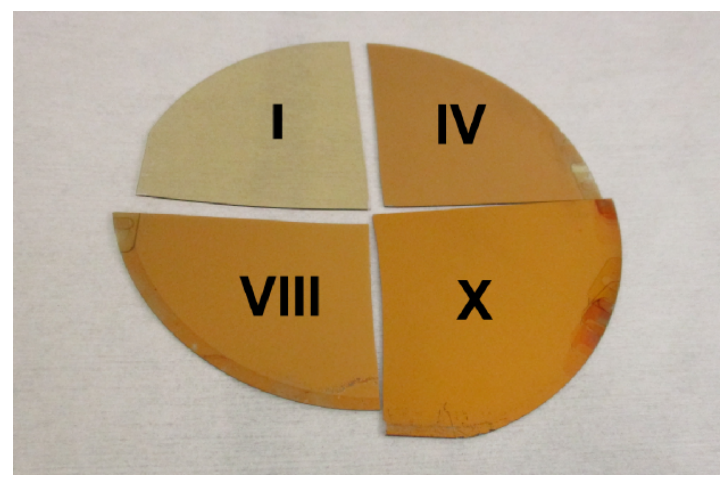

Fig. 2 Images of various HMM samples with 1, 4, 8 and 10 periods denoted by the roman numerals. Each sample is a quarter of a 4 -inch wafer in size.

To carry out the experiment, different HMM samples having 10 $\mathrm{nm}: 10 \mathrm{~nm}\left(\mathrm{Au}: \mathrm{Al}_{2} \mathrm{O}_{3}\right)$ as a unit cell were fabricated. Each sample had different number of periods, from 1 to 10, on 4 inch glass wafers as shown in Figure 2. Due to the poor adhesion between the oxide and noble metals, we used an APTMS adhesion layer in between the $\mathrm{Au}$ and $\mathrm{Al}_{2} \mathrm{O}_{3}$ to obtain smooth and continuous films. The fabrication routine consisting of a combination of two deposition techniques, sputtering for $\mathrm{Au}$ and atomic layer deposition for $\mathrm{Al}_{2} \mathrm{O}_{3}$, was used to maintain a roughness as low as possible, thus keeping it below $1 \mathrm{~nm}$ even after the $10^{\text {th }}$ period. The details of the fabrication procedures of the HMMs are presented in the HMM Fabrication under Methods Section.

We investigated the surface morphology of the Au nanofilms for each period using atomic force microscope (AFM). The surface roughness of each Au layer is shown in Table 2. The roughness

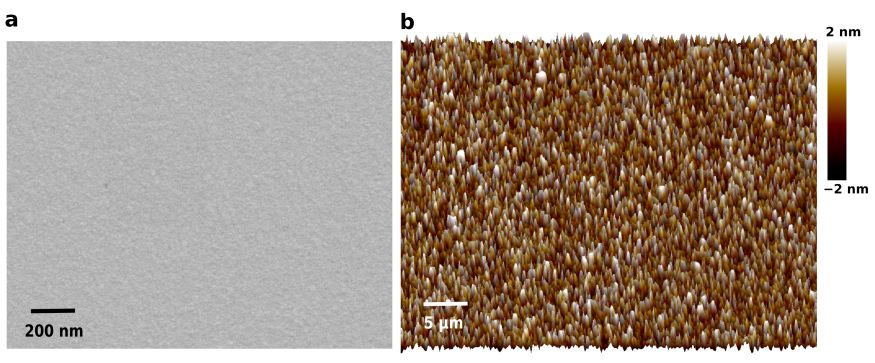

Fig. 3 Topological characterization of the $10^{\text {th }}$ period Au layer of the HMM. (a) SEM image showing a continuous layer with no interruptions. (b) AFM image for quantifying the roughness.

of the multilayer sample increases with the number of periods thus putting a practical limit on the maximum number of periods achievable. The films will form pin holes and defects if the roughness is more than 1 . The scanning electron microscope (SEM) and AFM images of the final $10^{\text {th }}$ period Au layer are shown in Figure 3a-b. The images confirm that the Au layer is continuous without any defects or pin holes. The final Au layer has a RMS roughness of $0.80 \mathrm{~nm}$. The increase in the surface roughness from $8^{\text {th }}$ period to $10^{\text {th }}$ period is due to the accumulation of roughness by more periods. Since the roughness is below $1 \mathrm{~nm}$ in this work, the effect of scattering is not visible in the optical characterization of the samples even after $10^{\text {th }}$ period.

Table 2 RMS roughness of Au layers of various periods of the HMM

\begin{tabular}{llllllll}
\hline Periods & 1 & 2 & 3 & 4 & 6 & 8 & 10 \\
\hline Roughness (nm) & 0.30 & 0.32 & 0.36 & 0.40 & 0.50 & 0.54 & 0.80 \\
\hline
\end{tabular}

We conducted a series of reflection measurements on structures with different number of periods using the Otto configuration setup with a TM-polarized light as schematically described in Figure 1. We used TM-polarized light to excite the plasmon modes supported by the multilayer hyperbolic metamaterial. On top of the HMMs we sputtered a $50 \mathrm{~nm} \mathrm{SiO} 2$ layer to maintain the symmetry of the modes and also to allow for excitation of high order modes. TM-polarized light is used to excite the modes. This way, we can identify the SPP-type modes and analyze their behavior. The reflection measurements are shown in Figure 4d-f and Figure $4 \mathrm{j}-1$ for periods $1,2,3$ and 4,8 and 10 , respectively. The noise close to $1100 \mathrm{~nm}$ visible in the experimental dispersion diagram is due to normalization errors arising from the spectral characteristics of the light source and not related to sample's response.

We performed 3D simulations of the reflectance of the multilayer HMMs of the various periods as described in Numerical Simulations in Methods section to compare with the experimental reflection measurements. In our 3D simulations, we also considered the APTMS adhesion layers. The simulation results are shown in Figure 4a-c for the periods 1, 2, 3 and Figure 4g-i for 4,8 , and 10 , respectively.

For the first period as shown in Figure 4 (a,d), the low reflection region beneath $35^{\circ}$ is due to the violation of total internal reflection (TIR) condition between the ZnSe prism and $50 \mathrm{~nm}$ $\mathrm{SiO}_{2}$ layer. For single period structure, the experimental reflec- 


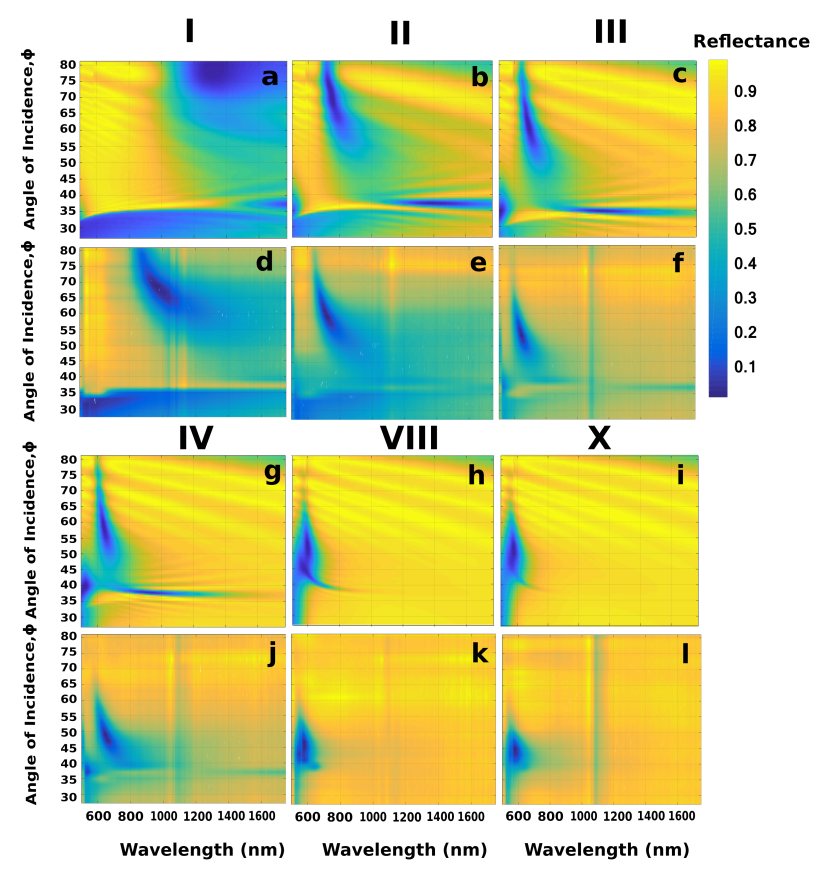

Fig. 4 Dispersion diagram of $\mathrm{HMM}$ modes for $\mathrm{ZnSe}$ prism- $\mathrm{SiO}_{2}(50$ $\mathrm{nm})-\mathrm{HMM}-\mathrm{SiO}_{2}(500 \mu \mathrm{m})$ structures. 3D EMA simulations (a-c),(g-i) and measurements (d-f),(j-l) with HMMs of periods 1, 2, 3 and 4, 8, 10, respectively. The roman numerals represent the number of periods of the HMM.

tion dip is observed at lower wavelengths between $900-1100$ $\mathrm{nm}$ for the same angle of incidence between $60^{\circ}$ to $80^{\circ}$ as compared to the broad reflection dip between 1300 - $1600 \mathrm{~nm}$ from simulation. This leads to a mean square reflection difference between effective medium prediction and experimental data to be 16\% MSDR. We used MSDR to calculate the reflection difference between simulation and experiment as defined in numerical simulations as shown in Table 3. The MSDR between experiment and simulation in Table 3 was calculated by

$$
M S D R=\sqrt{\frac{1}{N} \sum_{\phi, \lambda}\left[R(\lambda, \phi)_{\text {experimental }}-R(\lambda, \phi)_{\text {simulation }}\right]^{2}},
$$

where $\phi=27^{\circ}-81^{\circ}$ and $\lambda=500-1750 \mathrm{~nm}$ and $R(\lambda, \phi)_{\text {experimental }}$, $R(\lambda, \phi)_{\text {simulation }}$ are the experimental and simulation reflectance spectra respectively. $N=28028$ is the number of points used in this case.

With increasing the number of periods, the reflection dip at long wavelengths blue shifts and a new mode appears at short wavelengths as shown in Figure 4 (b, c, e, f). This new mode red shifts and this tendency is consistent in both experiments and theory. However, before reaching a thickness equivalent to four periods the simulations consistently show the long wavelength mode red shifted compared to experiments.

From the fourth period onwards, the reflection dips in experiment and simulation start to match very well, within 4\% MSDR. The reflection diagram on the $8^{\text {th }}$ period also shows that the two modes start to merge, and they completely overlap for the $10^{\text {th }}$

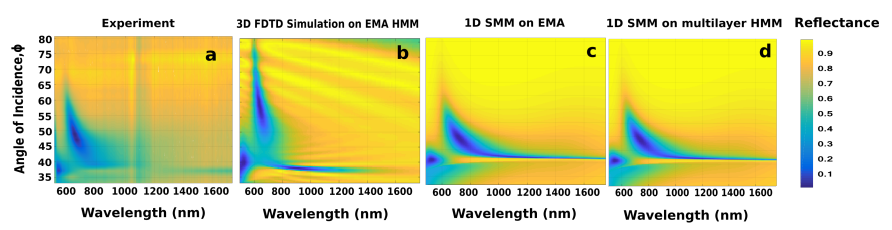

Fig. 5 Dispersion diagram of $\mathrm{HMM}$ modes for $\mathrm{ZnSe}$ prism- $\mathrm{SiO}_{2}(50$ $\mathrm{nm})-4^{\text {th }}$ Period $\mathrm{HMM} \mathrm{SiO}_{2}(500 \mu \mathrm{m})$ structures. (a) Experiment (b) 3D FDTD simulation on EMA HMM (c) 1D Scattering Matrix Method (SMM) on EMA and (d) 1D SMM on multilayer HMM, respectively.

period.

Table 3 Table of mean square error reflectance between 3D EMA simulation and experiment for the various periods of the HMM.

\begin{tabular}{lllllll}
\hline Periods & 1 & 2 & 3 & 4 & 8 & 10 \\
\hline 3D EMA vs Experiment & 15.9 & 5.5 & 5 & 3.6 & 3.8 & 3.5 \\
\hline $\begin{array}{l}\text { 1D SMM on multilayer structures } \\
\text { vs Experiment }\end{array}$ & 4.5 & 3 & 4 & 1.5 & 1 & 1.1 \\
\hline
\end{tabular}

In the calculation for reflectance of 1D SMM on multilayer HMM, we considered APTMS layers in Table 3. From Table 3, it can be observed that the reflection difference of the experimental and 3D EMA simulations decreases from $15.9 \%$ ( $1^{\text {st }}$ period) to $3.5 \%$ MSDR ( $10^{\text {th }}$ period) with increasing number of periods. This supports the prediction that the effective medium approximation starts to be accurate enough from a minimum number of periods. When comparing the accurate SMM theory with experimental results, one can see that, although generally decreasing, the difference between the spectra is maximum 4.5\% MSDR. Since the 1D SMM simulations are assuming the complete geometry of the structure we can consider that the maximum 4.5\% MSDR is the worst case scenario error, a combination between the experimental errors and the uncertainties in the modelling. Therefore, we can consider this number as a threshold for determining the applicability of the 3D EMA simulations. This leads us to state that the 3D EMA simulations are applicable only after the $4^{\text {th }}$ period. This conclusion further supports the one obtained from Table 1.

One should note in Figure 5 that there is a clear difference between the 1D simulation results used for the theoretical study and the 3D simulation and the experimental ones. Therefore, it is more relevant to use $3 \mathrm{D}$ simulations to compare with the experimental data, while the 1D SMM simulations may be used for comparing the two theoretical approaches.

\subsection{HMM Field Profile}

To further study the experimentally observed optical modes of the structure, we investigated the field profiles from the reflection dips of various periods of the HMM.

Figure 6 shows the real part of the tangential component of the transverse magnetic field profile at the reflection dips with the angle of incidence from $33^{\circ}$ to $85^{\circ}$ for various periods in the structure: 2, 4 and 10 respectively calculated with transfer matrix method as discussed in Numerical Simulations under Meth- 


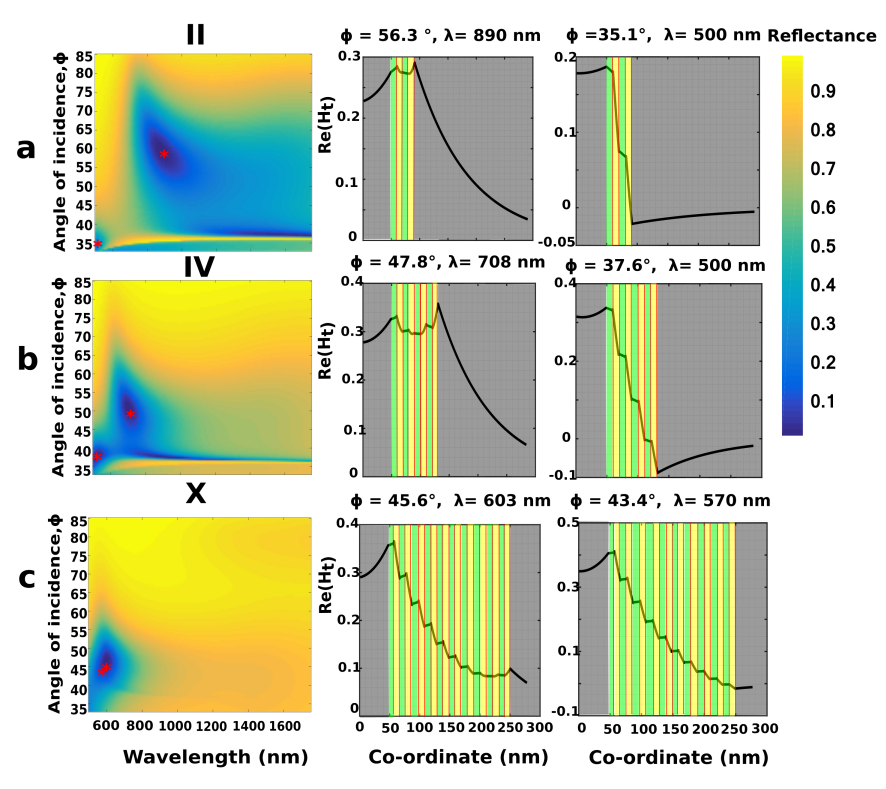

Fig. 6 Transverse magnetic field profile of the HMM for (a) 2, (b) 4, (c) 10 periods where the red asterisk $\left(^{*}\right)$ marks the reflection dips corresponding to the angle, $\phi$ and wavelength, $\lambda$ of the HMM dispersion diagram . The HMM is represented by the yellow and green color for $\mathrm{Au}$ and $\mathrm{Al}_{2} \mathrm{O}_{3}$ respectively and the red color represents the APTMS adhesion layer.The grey area represents the $\mathrm{SiO}_{2}$ layer beneath and above the HMM. The roman numerals represent the period of the HMM.

ods section. From Figure $6 a-c$ we can observe that symmetrical mode appears at higher angles and anti-symmetric mode at lower angles. The modes are due to the existence of metaldielectric-metal (MDM) coupling configuration over dielectricmetal-dielectric (DMD) one ${ }^{38}$. For the case of second period as shown in Figure 6a, the reflection dips observed at $\left(\phi=35.1^{\circ}, \lambda\right.$ $=500 \mathrm{~nm})$ and $\left(\phi=56.3^{\circ}, \lambda=890 \mathrm{~nm}\right)$ are related to modes that are anti-symmetrically coupled and symmetrically coupled surface plasmon polaritons respectively.

On the $4^{\text {th }}$ period, the modes at $\left(\phi=37.6^{\circ}, \lambda=500 \mathrm{~nm}\right)$ and $\left(\phi=47.8^{\circ}, \lambda=708 \mathrm{~nm}\right)$ starts to blue and red shift respectively as shown in Figure $6 \mathrm{~b}$. We could not observe any higher index modes of the HMMs which are related to the coupling of SPPs at the interfaces of the multilayer HMM in DMD configuration as analyzed by Higuchi et.al. ${ }^{39}$. This is because the maximum effective mode index achievable in this configuration is about 2.5, the $\mathrm{ZnSe}$ refractive index.

For the $10^{\text {th }}$ period as shown in Figure $6 \mathrm{c}$, the two modes observed at $\left(\phi=45.6^{\circ}, \lambda=603 \mathrm{~nm}\right)$ and $\left(\phi=43.4^{\circ}, \lambda=570 \mathrm{~nm}\right)$ merge into one.

\section{Methods}

\subsection{HMM Fabrication}

The HMM samples were fabricated on $500 \mu \mathrm{m}$ thick glass wafers. The wafers were precleaned in Piranha solution $\left(70 \% \mathrm{H}_{2} \mathrm{SO}_{4}+\right.$ $30 \% \mathrm{H}_{2} \mathrm{O}_{2}$ ) for 20 minutes to remove any organic dust residues. The unit cell consist of four-layers: 1nm APTMS - 10nm Au - 1nm APTMS - $10 \mathrm{~nm} \mathrm{Al}_{2} \mathrm{O}_{3}$. Fabrication starts with the immersion of the cleaned wafers into $2.5 \%$ APTMS in isopropyl alcohol (IPA) solution for 3 hours ${ }^{40}$ to deposit the APTMS adhesion layer. Then $10 \mathrm{~nm} \mathrm{Au}$ layer was sputtered at 2 mtorr pressure with a deposition rate of $10 \AA$ /second. Another layer of APTMS is deposited on top of the $10 \mathrm{~nm}$ Au layer in the similar way before the deposition of $\mathrm{Al}_{2} \mathrm{O}_{3}$. The deposition of $10 \mathrm{~nm} \mathrm{Al}_{2} \mathrm{O}_{3}$ was done using atomic layer deposition at temperature of $200{ }^{\circ} \mathrm{C}$ for 93 cycles where each cycle consists of 0.1 second pulse time of the precursors trimethyl-aluminum (TMA) and $\mathrm{H}_{2} \mathrm{O}$. We continued fabrication of various samples with different periods following the same process steps.

\subsection{Optical Characterization}

The reflection measurements were performed using high index ZnSe semi-cylinder prism of refractive index ranging from 2.4 to 2.7 in the visible and near infrared wavelength regime. TMpolarized light was used to excite the modes. The light source was a Super-K high continuum broadband laser from NKT Photonics allowing us to measure from a wavelength of $500 \mathrm{~nm}$ to $1750 \mathrm{~nm}$. The samples were placed on the ZnSe prism with the help of a tightening screw with an unavoidable air gap between the sample and the prism. The incidence angles were varied from $27^{\circ}$ to $81^{\circ}$ with a $2^{\circ}$ interval by rotating the mechanical stage. Each incidence angle was measured five times when ascending and five times when descending the angle to avoid the mechanical stage uncertainties. The reference spectrum is the measure of the reflectance without the sample at $45^{\circ}$ incidence. Note that the noise close to $1100 \mathrm{~nm}$ visible in the experimental dispersion diagram is due to normalization errors arising from the spectral characteristics of the light source and not related to sample's response.

\subsection{Numerical Simulations}

The series of calculation in Table 1 were performed by the scattering matrix formalism to calculate the reflectance from the multilayer structures ${ }^{41}$. We considered TM-polarized incident light with the magnetic field parallel to the plane of interface and for the same range of wavelengths of $\lambda=500-1750 \mathrm{~nm}$ and the angle of incidence as in the experiment, $\phi=27^{\circ}-81^{\circ}$ for the case of calculation in Table 1 and $\phi=33^{\circ}-85^{\circ}$ for the simulation in Figure 6.

The model structure consists $\mathrm{ZnSe}$ prism- $\mathrm{SiO}_{2}$ (50 nm thick)$\mathrm{HMM}-\mathrm{SiO}_{2}$ substrate. Here $\mathrm{HMM}$ is either multilayer of $\mathrm{Au}$ and $\mathrm{Al}_{2} \mathrm{O}_{3}$ or the one characterized by EMA.

According to EMA ${ }^{18}$, the effective ordinary and extraordinary permittivities, $\varepsilon_{o}$ and $\varepsilon_{e}$ are expressed by

$$
\begin{aligned}
\varepsilon_{o} & =f_{m} \cdot \varepsilon_{m}+f_{d} \cdot \varepsilon_{d} \\
\varepsilon_{e} & =\left(\frac{f_{m}}{\varepsilon_{m}}+\frac{f_{d}}{\varepsilon_{d}}\right)^{-1},
\end{aligned}
$$

where $\varepsilon_{m}$ and $\varepsilon_{d}$ are the permittivities of metal and dielectric, and $f_{m}$ and $f_{d}$ are the volume fraction of metal and dielectric layers, respectively. In general, EMA is assumed to hold under the condition that the thicknesses of each layer are deeply subwavelength. We note that in our structure with thickness of 10 
$\mathrm{nm}$ for both $\mathrm{Au}$ and $\mathrm{Al}_{2} \mathrm{O}_{3}$ layers as compared to the wavelength interval of $\lambda=500-1750 \mathrm{~nm}$, we maintain the ratio between the period thickness $(\Lambda=20 \mathrm{~nm})$ and wavelengths of $\Lambda / \lambda=1 / 25-$ $1 / 87.5$. The permittivity of the $\mathrm{Al}_{2} \mathrm{O}_{3}$ film, $\varepsilon_{d}$, was measured by ellipsometer. The permittivity of Au film, $\varepsilon_{m}$, is characterized by the Drude-Lorentz model: ${ }^{42}$

$$
\varepsilon_{m}=1+\sum_{m=0}^{m=5} G_{m} \Omega_{m}^{2} /\left(\omega_{m}^{2}-\omega^{2}+j \omega \Gamma_{m}\right)
$$

where $m=0$ is the Drude term, thus $\omega_{0}=0$.

The permittivities of $\mathrm{ZnSe}$ prism and fused silica $\left(\mathrm{SiO}_{2}\right)$ substrate are taken from Connolly et al. ${ }^{43}$ and Malitson et al. ${ }^{44}$ respectively.

Reflectance spectra of the effective medium approximated HMMs, provided in Figure 4, are conducted with use of commercially available Lumerical software. To compare more precisely with the experimental results, we included APTMS adhesion layer in the unit cell and used 3D FDTD simulations. The refractive index of APTMS is assumed to be $1.46 .^{45-47}$.

Here the HMM is an indefinite uniaxial layer homogenized by EMA for a unit cell of 4 layers with three different materials, APTMS - $\mathrm{Au}$ - APTMS - $\mathrm{Al}_{2} \mathrm{O}_{3}$, based on the below formula where $f$ parameters stand for volume fractions of $\mathrm{Al}_{2} \mathrm{O}_{3}$ as $f_{d_{1}}$, APTMS as $f_{d_{2}}$ and $\mathrm{Au}$ as $f_{m}$ in the unit cell.

$$
\begin{gathered}
\varepsilon_{o}=f_{m} \cdot \varepsilon_{m}+f_{d_{1}} \cdot \varepsilon_{d 1}+f_{d_{2}} \cdot \varepsilon_{d_{2}} \\
\varepsilon_{e}=\left(\frac{f_{m}}{\varepsilon_{m}}+\frac{f_{d_{1}}}{\varepsilon_{d_{1}}}+\frac{f_{d_{2}}}{\varepsilon_{d_{2}}}\right)^{-1}
\end{gathered}
$$

A nested parameter sweep is used to calculate the reflection of TM-polarized plane wave for angles between $27^{\circ}-81^{\circ}$ and wavelengths between 500 - $1750 \mathrm{~nm}$ for different thickness of homogenized HMM corresponding to 1, 2, 3, 4, 8, and 10 periods.

The transverse magnetic field profile in Figure 6 is calculated using transfer-matrix method ${ }^{48}$. We use the reflectance coefficient to obtain the tangential field components at ZnSe prism$\mathrm{SiO}_{2}$ interface. This obtained field was further used to calculate the fields in structure, considering $1 \mathrm{~nm}$ resolution.

\section{Conclusions}

High quality periodic multilayer structures with number of periods from 1 to 10 were fabricated and optically characterized using prism coupling experiment. The study was to compare reflectance by the various structures with the one predicted by the effective medium approximation. The structures were fabricated by combining two deposition techniques: sputtering and atomic layer deposition which allows controlling the thickness and minimizing the roughness of each layer of the HMMs. The final RMS roughness obtained was $0.80 \mathrm{~nm}$. The optical characterization also shows a very good agreement with the effective medium approximation calculation using 3D FDTD method as close as below $4 \%$ MSDR in comparison to the experiment from the $4^{\text {th }}$ period of the structure. Our findings show that we need at least 4 periods of the multilayer structure to comply with the criteria for the applicability of EMA.

\section{Conflicts of interest}

There are no conflicts to declare.

\section{Acknowledgement}

This research was supported by Villum Fonden "DarkSILD project No. 11116", and Direktør Ib Henriksens Fond, Denmark. Theoretical analysis was carried out with the support of the Ministry of Education and Science of the Russian Federation (3.1668.2017/4.6) and the grant of the President of the Russian Federation (MK-403.2018.2). Modeling was carried out with the support of the Ministry of Education and Science of the Russian Federation (3.1668.2017/4.6). All the fabrication work was performed at DTU Danchip.

\section{References}

1 A. Poddubny, I. Iorsh, P. Belov and Y. Kivshar, Nature Photonics, 2013, 7, 948-957.

2 E. E. Narimanov and A. V. Kildishev, Nature Photonics, 2015, 9, 214-216.

3 Z. Jacob, J. Y. Kim, G. V. Naik, A. Boltasseva, E. E. Narimanov and V. M. Shalaev, Applied Physics B: Lasers and Optics, 2010, 100, 215-218.

4 D. Lu, J. J. Kan, E. E. Fullerton and Z. Liu, Nature nanotechnology, 2014, 9, 48-53.

5 D. Lu, H. Qian, K. Wang, H. Shen, F. Wei, Y. Jiang, E. E. Fullerton, P. K. L. Yu and Z. Liu, Advanced Materials, 2018, 1706411, 1-7.

6 Z. Liu, H. Lee, Y. Xiong, C. Sun and X. Zhang, Science, 2007, 315, 1686.

7 J. Sun and N. M. Litchinitser, ACS Nano, 2018, 12, 542-548.

8 D. Lu and Z. Liu, Nature Communications, 2012, 3, 1205.

9 K. V. Sreekanth, Y. Alapan, M. ElKabbash, E. Ilker, M. Hinczewski, U. A. Gurkan, A. De Luca and G. Strangi, Nature Materials, 2016, 15, 4-11.

10 F. Wu, G. Lu, Z. Guo, H. Jiang, C. Xue, M. Zheng, C. Chen, G. Du and H. Chen, Physical Review Applied, 2018, 10, 064022.

11 K. V. Sreekanth, Y. Alapan, M. ElKabbash, E. Ilker, M. Hinczewski, U. A. Gurkan, A. De Luca and G. Strangi, Nature Materials, 2016, 15, 621-627.

12 T. Galfsky, Z. Sun, C. R. Considine, C. T. Chou, W. C. Ko, Y. H. Lee, E. E. Narimanov and V. M. Menon, Nano Letters, 2016, 16, 4940-4945.

13 P. Guo, B. T. Diroll, W. Huang, L. Zeng, B. Wang, M. J. Bedzyk, A. Facchetti, T. J. Marks, R. P. H. Chang and R. D. Schaller, ACS Photonics, 2018, 5, 2000-2007.

14 O. Takayama, E. Shkondin, A. Bodganov, M. E. Aryaee Panah, K. Golenitskii, P. Dmitriev, T. Repän, R. Malureanu, P. Belov, F. Jensen and A. V. Lavrinenko, ACS Photonics, 2017, 4, 28992907.

15 E. Shkondin, T. Repän, M. E. Aryaee Panah, A. V. Lavrinenko and O. Takayama, ACS Applied Nano Materials, 2018, 1, 1212-1218. 
16 O. Takayama, P. Dmitriev, E. Shkondin, O. Yermakov, M. Panah, K. Golenitskii, F. Jensen, A. Bogdanov and A. Lavrinenko, Semiconductors, 2018, 52, 442-446.

17 A. V. Kabashin, P. Evans, S. Pastkovsky, W. Hendren, G. A. Wurtz, R. Atkinson, R. Pollard, V. A. Podolskiy and A. V. Zayats, Nature Materials, 2009, 8, 867-871.

18 L. Ferrari, C. Wu, D. Lepage, X. Zhang and Z. Liu, Progress in Quantum Electronics, 2015, 40, 1-40.

19 C. L. Cortes, W. Newman, S. Molesky and Z. Jacob, Journal of Optics, 2014, 16, 063001.

20 J. Kim, V. P. Drachev, Z. Jacob, G. V. Naik, A. Boltasseva, E. E. Narimanov and V. M. Shalaev, Optics Express, 2012, 20, 81008116.

21 M. Y. Shalaginov, S. Ishii, J. Liu, J. Liu, J. Irudayaraj, A. Lagutchev, A. V. Kildishev and V. M. Shalaev, Applied Physics Letters, 2013, 102, 173114.

22 A. Kossoy, V. Merk, D. Simakov and S. A. M. Leosson, Stéphane Kéna-Cohen, Advanced Optical Materials, 2015, 3, 71-77.

23 R. Malureanu and A. Lavrinenko, Nanotechnology Reviews, 2015, 4, 259-275.

24 M. Todeschini, A. Bastos Da Silva Fanta, F. Jensen, J. B. Wagner and A. Han, ACS Applied Materials and Interfaces, 2017, 9, 37374-37385.

25 T. G. Habteyes, S. Dhuey, E. Wood, D. Gargas, S. Cabrini, P. J. Schuck, A. P. Alivisatos and S. R. Leone, ACS Nano, 2012, 6, 5702-5709.

26 J. Sukham, O. Takayama, A. V. Lavrinenko and R. Malureanu, ACS Applied Materials \& Interfaces, 2017, 9, 25049-25056.

27 S. V. Zhukovsky, O. Kidwai and J. E. Sipe, Optics Express, 2013, 21, 14982.

28 A. A. Bogdanov and R. A. Suris, JETP Letters, 2012, 96, 49-55.

29 O. Kidwai, S. V. Zhukovsky and J. E. Sipe, Physical Review A, 2012, 85, 053842.

30 T. Li and J. B. Khurgin, Optica, 2016, 3, 1388.

31 T. Tumkur, Y. Barnakov, S. T. Kee, M. A. Noginov and V. Liberman, Journal of Applied Physics, 2015, 117, 103104.
32 G. Lu, F. Wu, M. Zheng, C. Chen, X. Zhou, C. Diao, F. Liu, G. Du, C. Xue, H. Jiang and H. Chen, Optics Express, 2019, 27, 5326.

33 S. Ishii, M. Y. Shalaginov, V. E. Babicheva, A. Boltasseva and A. V. Kildishev, Optics Letters, 2014, 39, 4663.

34 V. E. Babicheva, M. Y. Shalaginov, S. Ishii, A. Boltasseva and A. V. Kildishev, Optics Express, 2015, 23, 31109-31119.

35 S. Liang, C. Jiang, Z. Yang, D. Li, W. Zhang, T. Mei and D. Zhang, Journal of Optics, 2018, 20, 065001.

36 X. Yang, J. Yao, J. Rho, X. Yin and X. Zhang, Nature Photonics, 2012, 6, 450-454.

37 Z. Liu, H. Lee, Y. Xiong, C. Sun and X. Zhang, Science, 2007, 315, 1686.

38 G. Rosenblatt and M. Orenstein, Optics Express, 2011, 19, 20372.

39 M. Higuchi and J. Takahara, Optics Express, 2018, 26, 1918.

40 L. Leandro, R. Malureanu, N. Rozlosnik and A. Lavrinenko, ACS Applied Materials and Interfaces, 2015, 7, 5797-5802.

41 D. Y. K. Ko and J. R. Sambles, Journal of the Optical Society of America A, 1988, 5, 1863.

42 Optiwave, OptiFDTD Technical Background and Tutorials, Version 8.1, 2009, 27.

43 J. Connolly, B. DiBenedetto and R. Donadio, Proc. SPIE, 1979, pp. 141-144.

44 I. H. Malitson, Journal of the Optical Society of America, 1965, 55, 1205.

45 L. Brigo, E. Gazzola, M. Cittadini, P. Zilio, G. Zacco, F. Romanato, A. Martucci, M. Guglielmi and G. Brusatin, Nanotechnology, 2013, 24, 155502.

46 M. Terracciano, I. Rea, J. Politi and L. De Stefano, Journal of the European Optical Society, 2013, 8, 1-6.

47 L. De Stefano, G. Oliviero, J. Amato, N. Borbone, G. Piccialli, L. Mayol, I. Rendina, M. Terracciano and I. Rea, Journal of The Royal Society Interface, 2013, 10, 20130160-20130160.

48 M. Born and E. Wolf, Principles of Optics 7th edition, 1999, pp. $1-952$. 